095

\section{SIGNIFICATO DIVCA IgG ISOLATE IN CORSO DI INFEZIONE DA EBV}

Clerici P., De Paschale M., Agrappi M., Belvisi L., Cagnin D., Cerulli T., Manco M.T., Marinoni L., Mirri P., Paganini A.

\section{U.O. Microbiologia, A.O. Ospedale Civile di Legnano (Mi)}

Introduzione. Il quadro sierologico rappresentato da positività per VCA IgG e negatività per VCA IgM e EBNA crea problemi interpretativi nell'inquadramento dell'infezione da EBV. Scopo del nostro lavoro è stato di valutare l'entità del fenomeno e di interpretarne il significato.

Materiali e metodi. Sono stati esaminati per la ricerca di VCA IgG, IgM e EBNA 2422 campioni di siero (1178 maschi e 1244 femmine, età 0-92 anni) di pazienti afferenti per un controllo clinico all’U.O. Microbiologia dell'Ospedale di Legnano.

Risultati. 177 campioni (7.31\%) sono risultati VCA IgG positivi e VCA IgM e EBNA negativi. Di questi, 13 (7.34\%) sono risultati positivi al MonoTest.

La prevalenza nelle varie classi di età varia dal $4.5 \%$ della classe d'età 0-10 anni all' $11.0 \%$ nella classe $51-60$, senza differenza tra maschi e femmine.

Per 35 soggetti (di cui 7 con MonoTest positivo) è stato possibile ottenere un controllo a distanza di tempo.

Dopo tre settimane, dei 7 soggetti MonoTest positivi, il pattern anticorpale è rimasto invariato in 3 soggetti, mentre altri 3 risultavano positivi per VCA IgG e IgM (negativi per EBNA), e 1 positivo per VCA IgG e IgM e EBNA. Dei 28 soggetti MonoTest negativi il pattern anticorpale è rimasto invariato per 25 soggetti dopo tre settimane (per 10 di questi il pattern è rimasto invariato anche dopo 180 giorni e per 1 dopo 360 giorni), mentre 3 sono risultati positivi per VCA IgG e EBNA ad un controllo eseguito rispettivamente dopo 39, 100 e 202 giorni.

Conclusioni. Nella nostra casistica il profilo anticorpale in studio si presenta nel 7\% circa dei casi. Nella maggioranza dei casi con MonoTest negativo questo profilo rimane costante nel tempo indicando un'infezione pregressa, mentre in oltre la metà dei casi con MonoTest positivo la comparsa nel tempo di VCA IgM depone per un'infezione acuta.

\section{6 \\ RILEVAZIONE DI VIRUS RESPIRATORI MEDIANTE TECNICHE TRADIZIONALI E MOLECOLARI IN PAZIENTI PEDIATRICI}

\author{
Coato P'., Boaretti M.',2, Pegoraro M²., Fontana R..,2 \\ 'Dipartim. di Patologia, Sez. di Microbiologia, Università di Verona \\ 2Servizio di Microbiologia, Immunologia e Virologia, \\ Azienda Ospedaliera di Verona
}

Introduzione. Le infezioni acute delle vie respiratorie inferiori nell'età pediatrica sono causa di rilevante morbilità e mortalità. Lo scopo di questo studio è stato quello di valutare la prevalenza dei vari virus respiratori in bambini ospedalizzati per infezione acuta delle basse vie respiratorie, utilizzando sia tecniche tradizionali che tecniche di biologia molecolare.

Metodi. Gli aspirati naso-faringei provenienti da 110 pazienti pediatrici (età media: 12,8 mesi) ricoverati nel periodo dicembre 2005-maggio 2006 sono stati sottoposti ad immunofluorescenza diretta (DFA), esame colturale rapido a 24 e 72 ore ed esame colturale convenzionale per la ricerca dei seguenti virus: influenzale A e B, respiratorio sincinziale (RSV), parainfluenzale (PIV1-3) ed Adenovirus (ADV). Gli stessi campioni sono stati anche analizzati tramite PCR o RT-PCR per la ricerca degli stessi virus e inoltre per la determinazione della presenza del genoma dei Metapneumovirus (hMPV).

Risultati. Sono risultati positivi per la ricerca di virus respiratori in totale 63 campioni $(57,2 \%)$ dei quali 52 per RSV, 7 per Adenovirus e 4 per hMPV. I metodi tradizionali hanno evidenziato 45 campioni positivi per la ricerca di RSV, in 7 casi l'unica indagine positiva è stata l'RT-PCR. Per quanto riguarda la ricerca di Adenovirus 3 casi sono stati rilevati mediante esame colturale e 4 positività sono emerse dall'amplificazione genica.

Conclusioni. L'RSV si conferma essere una delle cause più frequenti di infezioni respiratorie acute nei bambini ospedalizzati. Le tecniche di biologia molecolare possono essere considerate un supporto per i metodi tradizionali molto utile nella rilevazione di virus respiratori, in particolare per quanto riguarda la ricerca di Adenovirus; nel caso dei hMPV l'approccio molecolare risulta essere fondamentale non essendo ancora disponibili tecniche immunologiche specifiche. 